\title{
Uso de la bicicleta en Costa Rica: repaso histórico y caracterización del tipo de ciclistas y su movilidad en el entorno vial nacional
}

\section{Use of bicycle in Costa Rica: historical review and characterization of the type of cyclists and their mobility on the national road system}

\section{Bohián Pérez Stéfanov}

Antropólogo-Investigador

Área de Investigación

Observatorio Costarricense de Seguridad Vial

Consejo de Seguridad Vial.

bohian@gmail.com

Fecha de recepción: 04 de agosto de 2017 / Fecha de aprobación: 01 de septiembre de 2017

\section{RESUMEN}

Son muy pocas las investigaciones sobre el uso de la bicicleta en Costa Rica. En los últimos años, dentro de los estudios de movilidad, transporte y seguridad vial, el tema del ciclismo ha ido ganando terreno como opción para la movilidad y posible solución al congestionamiento vial de las ciudades. Sin embargo, se ha desconocido y marginado aquellas localidades donde la bicicleta no solo es un medio de transporte de uso masivo consolidado históricamente, sino que forma parte íntegra de las estructuras sociales y económicas de las comunidades y sus dinámicas culturales.

Con la ampliación y mejoras de la red vial nacional, así como la implementación de políticas públicas que desconocen la importancia del uso de la bicicleta dentro de espacios sociales de alta complejidad, han generado un impacto directo y negativo en los patrones de movilidad de estas poblaciones, aumentando el riesgo de incidentes de tránsito.

Se proponen dos categorías para comprender el uso de la bicicleta: los ciclistas utilitarios, grupo heterogéneo cuyo elemento en común es el uso cotidiano de la bicicleta desde el espacio simbólico de sus comunidades y realidades históricas, muchas de ellas rurales; y el del ciclista recreativo, más homogéneo y con características más específicas, común a espacios urbanos y con un uso de la bicicleta más orientado al deporte no profesional y el esparcimiento.

PALABRAS CLAVE: Bicicleta, ciclismo, seguridad vial, movilidad segura, ciclistas utilitarios, ciclistas recreativos, cultura, sociedad.

\begin{abstract}
There is very few research on the use of bicycles in Costa Rica. Over the last years, the use of bicycles has been positioned throughout studies of mobility, transportation and road safety as an important mobility alternative and a possible solution to road traffic congestion in the cities. However, localities where the bicycle has not only been a historically consolidated means of transportation, but also an integral part of the social and economic structures of the communities and their cultural dynamics, they have been ignored and not been acknowledged.
\end{abstract}

With the construction and improvement of the National Road Network, and the implementation of public policies that do not consider the importance of the use of the bicycle within social spaces of high complexity, a direct and negative impact on the mobility patterns of these populations has been generated, increasing the risk of traffic accidents.

Two categories are proposed to understand the use of the bicycle: utilitarian cyclists, a heterogeneous group whose common element is the daily use of the bicycle from the symbolic space of their communities and historical realities, many of them on a rural context; and recreational cyclists, more homogenous and with more specific characteristics, common to urban spaces and with a bicycle use more oriented to nonprofessional sport and recreation.

KEYWORDS: Bicycle, cycling, road safety, safe mobility, utilitarian cyclist, recreational cyclist, culture, society. 


\section{INTRODUCCIÓN}

En el presente artículo se hace un breve repaso de la creación y evolución del velocípedo, entendido como aquel artefacto de una o más ruedas que funciona exclusivamente por el esfuerzo físico de una persona y que evolucionó en la actual bicicleta. $\mathrm{Su}$ redescubrimiento en los últimos años obedece a la creciente necesidad de redefinir los modelos de movilidad urbana dentro de las ciudades con redes viales cada vez más congestionadas y con calidad de vida cada vez menor por los efectos contaminantes de la flota automotor.

Lo anterior como referente para luego comprender el contexto histórico del uso de la bicicleta en Costa Rica, proponiendo la necesaria diferenciación entre lo que denomino ciclistas utilitarios y ciclistas recreativos; los primeros, como resultado de procesos históricos dentro de los cuales la bicicleta se ha convertido en una construcción simbólica que sobrepasa la dimensión de la propia movilidad, para pasar a ser parte integral del tejido social de comunidades y regiones con uso extendido de la bicicleta entre todos los segmentos de población; y los recreativos, como una tendencia de la última década en donde la bicicleta se consolida como opción para el esparcimiento y deporte de colectivos organizados. Estos últimos con características en común y cierta homogeneidad, frente a un ciclista utilitario con condiciones, necesidades, vulnerabilidades, riesgos y posibilidades muy heterogéneas.

La carencia de estudios científicos sobre el uso histórico de la bicicleta en Costa Rica, más allá del plano deportivo o de las propuestas sobre movilidad con perspectiva arquitectónico-ingenieril de los últimos años, hace de este artículo una primera aproximación que pretende abrir el debate y generar un mayor interés desde un enfoque integral, para comprender la complejidad del uso de la bicicleta y su vinculación a los procesos sociales, históricos, económicos y culturales de muchas comunidades del país.

Busca ampliar el debate sobre la implementación de nuevas obras viales y políticas públicas en seguridad vial, en aquellas regiones donde la bicicleta es de uso cotidiano con profundidad histórica, para así adaptar los procesos y la obra vial final a sus requerimientos, garantizando la movilidad segura de las personas y la consolidación de la bicicleta como alternativa para la movilidad sustentable.

La base para este artículo se desprende de las múltiples investigaciones en movilidad y seguridad vial desde un enfoque cualitativo y social que se han realizado desde el Área de Investigación y Estadísticas del Consejo de Seguridad Vial, con especial énfasis en algunos de los cantones con mayor arraigo en el uso de la bicicleta, como Osa (COSEVI, 2012), Puntarenas (COSEVI, 2013), Cañas y Liberia (COSEVI, 2014, 2017), Limón y Pococí (COSEVI, 2016), entre otros.

\section{ORIGEN Y EVOLUCIÓN DE LA BICICLETA MODERNA}

Cabe mencionar que algunas referencias buscan el origen del velocípedo (todo vehículo de una o más ruedas que se impulsa exclusivamente por la fuerza humana) entre algunas de las primeras grandes civilizaciones de la historia, como Egipto y China (Sáenz, 2014), o en diseños propuestos por algunos de los principales pensadores del Renacimiento europeo (Bijker, 1995; Sáenz, 2014), sin embargo, para efectos de este artículo se analizará su evolución a partir de los diseños surgidos y consolidados en el siglo XIX en algunos países del occidente europeo, desde donde se maduró el concepto que aún hoy en día centra la representación de lo que es una bicicleta y su uso masivo como vehículo de transporte no motorizado (Bijker, 1995).

La mayor parte de la literatura coincide que la bicicleta, como aquel artilugio de dos ruedas impulsado por la fuerza mecánica de una persona, aparece por primera vez entre finales del siglo XVIII e inicios del siglo XIX, en los países del centro de Europa, primero como una excentricidad de ricos y nobles, para finalmente ser el vehículo de uso masivo entre la clase obrera de la Europa industrial (Bijker, 1995; Harlihy, 2004; Sáenz, 2014) que no solo revolucionó la movilidad en las urbes de inicios del siglo XX, sino también las relaciones sociales y dinámicas culturales en prácticamente la totalidad del mundo.

Se asume que el primer velocípedo de dos ruedas (pero aun sin pedales, ni cadena, ni frenos) fue inventado por el alemán Karl Christian Ludwing Drais alrededor de 1817, o, por lo menos fue en ese año que lo presentó en público, elaborada en madera con la intención de "mejorar el rendimiento del desplazamiento humano” (Sáenz, 2014) nombrándola Laufmaschin o Máquina de Correr.

Poco más de 20 años después, el escocés Kirkpatrik MacMillan (1839) construye el primer modelo de un velocípedo con un sistema de propulsión mecánica por medio de pedales bastante artesanales. En sus inicios, todos estos modelos presentaban ruedas en madera, hierro u otro material y era común que la rueda delantera fuese más grande que la trasera (Bijker, 1995; Harlihy, 2004; Sáenz, 2014).

En esos primeros años, la mayoría de personas y autoridades terminaron por rechazar estos modelos rudimentarios de bicicleta, entre otras cosas, por su excesivo costo, pero también, por el alto riesgo para las personas, convirtiéndose los accidentes por caídas y atropellos una novedad que ciudades de Europa, Estados Unidos e incluso de Asia, pasaron a restringir y multar (Sáenz, 2014).

Pero en la Europa victoriana de finales del siglo XIX, y a pesar de los riesgos, la bicicleta se popularizó y existían muchos modelos, algunos bastante estrafalarios, en su mayoría con una rueda más 
grande que la otra (para así aumentar el radio de desplazamiento), accionando el sistema de pedaleo montado únicamente sobre la rueda delantera.

Para la década de 1880 los inventores ingleses Henry Lawson y John Kemp Starley fueron perfeccionando lo que llegarían a ser los primeros modelos de bicicleta con dos ruedas de igual tamaño. En 1885 Starley patenta el sistema de pedaleo con cadena en la rueda trasera y frenos, lo que se llegaría a conocer como safety bicycle o bicicleta segura por su innovadora forma y distribución, contrarrestando los diseños con rueda delantera mucho más grande, permitiendo ubicar los pedales a una distancia segura del suelo. Se considera como el inspirador del modelo moderno de las bicicletas (Bijker, 2004; Sáenz, 2014; Harlihy, 2004).

En 1890 John Boyd Dunlop diseñó lo que se llegaría a convertir en las ruedas de neumático con cámara de aire, en aquel entonces a base de caucho y que se podían inflar, lo que revolucionó el uso y modelos de las bicicletas, reduciendo su peso y tamaño, tornándolas más seguras.

Las innovaciones del diseño, junto a la producción en línea y el uso de variadas materias primas en su elaboración, permitieron que el costo de la bicicleta se redujera paulatinamente (Bijker, 1995; Harlihy, 2004; Sáenz, 2014), aumentando su disponibilidad y uso entre la creciente clase obrera y público en general.

Este acumulado de innovaciones en el diseño permitió que para inicios del siglo XX se tuviera un modelo estándar de bicicleta moderna que se ha mantenido casi inalterado hasta la actualidad, caracterizándose por un marco central en forma de "diamante" o de rombo, también llamado doble triángulo, que se convierte en la estructura base sobre la cual se montan las demás partes (Harlihy, 2004; Carreon y otros, 2011).

Proponiendo una clasificación muy general e inicial para Costa Rica, podemos decir que predominan tres estilos de bicicletas: el primer estilo, con características para carretera o carrera, que mantienen gran parte de la estructura descrita, siendo su principal modificación el tener unas ruedas muy delgadas y algunas sin neumáticos.

El segundo estilo y quizás de los más popularizados, es el conocido como "montañera" o todo terreno, caracterizándose por tener una estructura más robusta y pesada, incluyendo ruedas mucho más gruesas. En ambos modelos se ubican diferentes sistemas de frenado, cambios y otras innovaciones.

En una tercera categoría, muy genérica y heterogénea, podemos ubicar una serie de bicicletas con diferentes diseños, tamaños y variables, con una estructura intermedia entre los dos modelos anteriores, con finalidad más bien de uso cotidiano o doméstico, que, si bien cuentan con todos los elementos ya descritos, pueden carecer de sistema de cambios o tener uno mucho más sencillo, lo mismo que el sistema de frenado. Presentan ruedas gruesas y asientos más anchos, pero con características muy básicas.

\section{USO HISTÓRICO DE LA BICICLETA EN EL MUNDO}

Históricamente la movilidad de las personas se lograba mediante el caminar del individuo o con la ayuda de animales de tiro. Pero siempre se ha tenido el impulso por desarrollar máquinas que permitieran la movilidad humana de una forma más independiente y con mayor rendimiento, superando las limitantes físicas del cuerpo (Bijker, 1995).

Aunque desde finales del siglo XVIII las máquinas de vapor y otros inventos irrumpieron en la vida cotidiana de las sociedades occidentales, se necesitaron más de ciento cincuenta años (hasta mediados del siglo XX), para que la humanidad en su conjunto sustituyera de forma masiva la tracción animal, por otra mecánica y automotor: el carro.

En los cien años que transcurrieron entre la primera locomotora a vapor (década de 1780) y el primer vehículo de combustión interna (década de 1880), la bicicleta se erigió como el primer vehículo de tracción no animal que modificó sustancialmente la forma de traslado y movilidad de poblaciones enteras, sobre todo en las grandes ciudades de Europa, Norteamérica y Asia, consolidándose hasta convertirse en el medio de transporte favorito (y único) para millones de personas, impactando el desarrollo de sus estructuras sociales y dinámicas culturales (Bijker, 1995). Aunque en sus inicios fue un artilugio accesible solo a los sectores de mayor poder económico de Europa (Sáenz, 2014), ya para inicios del siglo XX su uso estaba extendido por el mundo, impactando sobre todo las clases obreras de los países industrializados.

Su fácil maniobrabilidad, bajo costo de adquisición, economía en su uso, velocidad, durabilidad, mantenimiento y reparación sencilla, accesibilidad a prácticamente cualquier tipo de entorno, edad y sector social, apuntaló a la bicicleta como el primer gran reformador de las dinámicas de movilidad en el mundo, extendiéndose y adaptándose a las necesidades, requerimientos y usos variados de cada región y país, incluso de cada usuario (Herlihy, 2004; EC, 1999).

Con la irrupción de los vehículos automotores de menor costo a partir de la década de 1910, en conjunto con el auge de un sistema cultural de consumo al estilo occidental en las últimas cinco décadas; disminuyó el uso de la bicicleta pasando a ser más un objeto para el entretenimiento y deporte, que vehículo para la movilidad, sobre todo en los entornos urbanos y metropolitanos. Aunque su presencia se ha mantenido como rasgo distintivo en la 
movilidad de millones de personas en el mundo, particularmente en zonas rurales.

A pesar de los embates del modernismo en el consumo de vehículos automotores, la bicicleta resistió y no desapareció. Ante los nuevos paradigmas del pensamiento humano respecto a su impacto en el equilibrio ecológico del planeta, la bicicleta ha empezado a reinventarse como la única y mejor opción para la movilidad sustentable de las personas, sobre todo en las grandes urbes y ciudades, de donde metódicamente se intentó marginar (ITDP, 2012).

Sus calidades propias están siendo revalorizadas bajo el nuevo esquema de la huella ecológica que el ser humano está generando en el planeta con sus necesidades de transporte diario. El bajo costo, la cero emisión de cualquier tipo de gases o desechos, su versatilidad como vehículo para ciudades cada vez más estrechas, más el extra que implica el esfuerzo de la persona para su impulso (convertido en ejercicio físico dentro de sociedades cada vez con mayores índices de sedentarismo y obesidad), está logrando que la bicicleta como vehículo se ubique en el foco de atención de todo estudio de urbanismo, movilidad, seguridad vial y transporte que se estime serio y responsable (EC, 1999; ITDP, 2012).

En la actualidad, muchas de las principales metrópolis del mundo y ciudades con altos estándares de calidad de vida, tienen en la bicicleta uno de sus principales pilares, con impactos positivos y directos en la salud de la población, la movilidad, el ordenamiento urbano y la prevención de accidentes de tránsito (Fletsbearaad, 2010; EC, 1999).

\section{LA BICICLETA EN COSTA RICA}

Para Costa Rica no se pudieron identificar estudios o investigaciones concretas que rescaten la presencia de la bicicleta como medio de transporte en las dinámicas históricas y socioculturales del país o sus comunidades.

En términos generales, la presencia de la bicicleta en las investigaciones académicas se encuentra apenas en los últimos años, siguiendo la tendencia mundial por repensar los espacios urbanos y opciones de movilidad por medios sustentables y sostenibles que no contaminen ni impacten más los sistemas de tránsito ya de por sí colapsados.

Hay una importante referencia bibliográfica en cuanto a la práctica del ciclismo como deporte y deporte élite, incluso con algunas referencias en estudios de género, a través de las cuales se rescatan unos pocos datos históricos sobre la bicicleta en Costa Rica (Hughes, 2012; Ulloa, 2010). De ellas podemos rescatar algunas inferencias históricas.
Para finales del siglo XIX, muchas de las grandes ideas del modernismo, liberalismo y avances tecnológicos se impulsaban desde los países del Occidente como Francia, Inglaterra y Alemania, que como se señaló, desarrollaron las principales innovaciones del modelo actual de bicicleta.

Es en estos mismos países que muchos de los intelectuales costarricenses de la época e hijos de familias acomodadas fueron a realizar sus estudios o a participar de las redes mercantiles con las potencias europeas. Hay extensos estudios que demuestran la influencia de las ideas europeas de la época en el pensamiento liberal de la intelectualidad costarricense (Salazar, 1990; Morales, 1993; Molina, 2002; Quesada, 2007).

Por tanto, no resulta extraño suponer que una innovación como la bicicleta, que para la segunda mitad del siglo XIX era la moda en París y Londres, terminara por ser importada por los liberales intelectuales costarricenses a las calles del San José del siglo XIX, época en la cual el máximo exponente del transporte de personas y cargas era sin duda la yunta de bueyes y la carreta y los animales de tiro.

Es probable que las primeras bicicletas significaran un marcado contraste dentro del contexto de la ciudad de San José y sus calles en tierra; hay una referencia que para finales del siglo ubica en lo que es hoy el parque España, la práctica de carreras en bicicleta con la participación de algunas de las familias más influyentes de la época (Hughes, 2012).

El ciclismo como práctica profesional de élite siguió acaparando espacios hasta ser considerado de manera oficial como deporte y tener su primer campeonato nacional en la década de 1940. Eso sí, un deporte exclusivamente masculino. No fue hasta los Juegos Nacionales de 1990 que se dio la primera participación femenina en ciclismo profesional (Sánchez, 1991; Hughes, 2012).

Otros estudios publicados no se encontraron. Es así que en el marco de estudios de movilidad y riesgos en seguridad vial impulsados desde el Área de Investigación y Estadísticas del Consejo de Seguridad Vial a partir del año 2012 en cantones y regiones fuera del Valle Central como Osa (COSEVI, 2012), San Carlos, Puntarenas (COSEVI, 2013), Pococí, Limón (COSEVI, 2016), Liberia, Cañas (COSEVI, 2014, 2017), Pérez Zeledón (COSEVI, 2012), se empezaron a recabar datos e información que documentó la presencia de la bicicleta como medio de transporte, no solo para la movilidad de las personas en su cotidianidad, sino también en la construcción de complejas redes de socialización, llegando a convertirse la bicicleta en un elemento importante para comprender y explicar algunos de los procesos históricos, socioculturales y económicos de gran parte de las zonas rurales del país, vinculadas a algunas de las principales arterias viales 
como las rutas nacionales 1 y 2 (Interamericana), 21 (Nicoya), 23 (Barranca), 17 (Puntarenas), 32 (Limón) y 34 (Costanera).

Sobre el uso cotidiano de la bicicleta entre la población de Costa Rica fuera del Valle Central, no se ha podido encontrar una referencia directa, aunque parece ser que su extensión en el territorio obedece tanto a condiciones propios del terreno (y no tanto climáticos) como a factores culturales e históricos.

En cuanto a la topografía del terreno como categoría de análisis, se ha podido registrar y observar que hay una clara relación entre aquellas regiones con una geografía regular, sin abruptos cambios altitudinales, con extensas zonas llanas o con leves ondulaciones, con la presencia histórica del uso de la bicicleta en la cotidianidad de las poblaciones (Osa, Limón, Cañas, Puntarenas, entre otros); frente a aquellas otras regiones, más montañosas y accidentadas como las del Valle Central, en donde si bien se registra un uso histórico de la bicicleta, pareciera que su alcance fue mucho más limitado, o, fue más propensa a ser sustituida por otros medios de transporte.

Sobre los factores culturales e históricos, se pueden identificar algunos elementos comunes entre aquellos cantones con una preponderancia particular en el uso de la bicicleta como medio de transporte masivo. En principio, es común que sean zonas costeras y rurales (como Puntarenas, Quepos, Osa Limón, Sixaola), aunque no exclusivamente (como Cañas, Liberia, Santa Cruz o Nicoya) pero que en general tienen en el sector agropecuario y de monocultivos su principal actividad económica (banano, plátano, palma, caña, naranjas, piña), así como fuertes procesos migratorios de múltiples orígenes en diferentes momentos históricos.

Un hecho documentado que al parecer influyó en el cambio cultural en cuanto al uso de la bicicleta lo es el fuerte proceso migratorio de población asiática, principalmente de China, con un arraigo que muchas veces se debe buscar en los finales del siglo XIX, con los planes de construcción y ampliación de la red ferroviaria en el país o de los monocultivos de las grandes trasnacionales. La presencia de inmigrantes chinos en Puntarenas, Cañas, Limón y Ciudad Cortés ha sido analizada en varios estudios (Fonseca 1979; 2016; Loría, 2000).

A partir de tradiciones orales recabadas entre informantes mayores de Ciudad Cortés en Osa, en Cañas (Guanacaste) y en el centro de Puntarenas (cantón), fue común escuchar que los primeros recuerdos de bicicletas en las calles de esos pueblos de la primera mitad del siglo XX se remontan a comerciantes chinos y sus familias.

Quizás con estilos mucho más burdos que las bicicletas de la élite económica de San José, estos primeros vehículos rápidamente se empezaron a posicionar dentro de las dinámicas cotidianas de movilidad de las poblaciones locales, que por primera vez vieron en la bicicleta esa opción de transporte para distancias que antes se tenían que recorrer a pie y en mucho más tiempo. Esto sin duda generó un importante cambio cultural, que aún perdura y se puede reconocer entre sus poblaciones.

Para la segunda mitad del siglo XX, la bicicleta ya era un constructo permanente en los procesos sociales, económicos y culturales de las regiones costeras y rurales fuera del Valle Central. No solo como medio de transporte, sino como parte de las dinámicas socioculturales de las comunidades y sus habitantes, lo que se deduce del hecho que muchas personas con más de 70 años, aun hoy día guardan recuerdos de la presencia de la bicicleta desde sus épocas de infancia.

En la actualidad, en cualquiera de estos cantones, la bicicleta sigue siendo parte intrínseca de los procesos culturales y de socialización de sus poblaciones. Su uso masivo es fácilmente observable en todas sus calles, en las escuelas y colegios, instituciones públicas $\mathrm{y}$ en los fines de semana, entre todos los segmentos de sus pobladores y de todas las edades.

Se destaca que el clima no parece ser un factor determinante en la consolidación de la bicicleta como medio de transporte, por cuanto los cantones que registran un uso masivo de la misma, presentan también condiciones de alta humedad y temperatura, intensa radiación solar, así como fuertes y prolongadas temporadas de lluvia.

\section{ANÁLISIS SOBRE EL USO SOCIAL DE LA BICICLETA EN COSTA RICA}

En este apartado se propone una nomenclatura diferenciada para el análisis del uso de la bicicleta: por un lado, se definen a los ciclistas utilitarios, como aquellos usuarios tradicionales para quienes la bicicleta no es solo un medio de transporte, sino que cumple muchas otras funciones de alcance cultural y simbólico, predominando en espacios rurales; en otro grupo estarían aquellos que es común denominar como ciclistas recreativos, cuyo uso de la bicicleta se inserta dentro de una tendencia coyuntural del hacer deporte colectivo, en condiciones y con características muy distintas al ciclista utilitario, aunque muchas veces comparten los mismos espacios viales y sociales.

La idea central es aportar detalles en las características y diferencias de estos dos segmentos de usuarios de la bicicleta: el primero heterogéneo y variado, con elementos en común más bien simbólicos y con profundidad histórica; el segundo más homogéneo, de extracción temporal más reciente y de espacios más bien urbanos. Estas diferencias generan distintas necesidades 
para su movilidad segura, por tanto es fundamental tenerlas presente de previo a los procesos de construcción y planificación de la red vial del país y el equipamiento para la movilidad segura de las vías, incluyendo la construcción de ciclovías y/o ciclorutas.

\section{Uso de la bicicleta y dinámicas culturales en Costa Rica: el ciclista utilitario}

Todo proceso de movilidad entre una población responde tanto a necesidades económicas como a condiciones sociales, culturales y ambientales.

Para las localidades de Costa Rica en las que aún persiste un uso extendido de la bicicleta como medio de transporte, se ha podido identificar su impacto en la modelación de los patrones de movilidad, pero también en el desarrollo de las relaciones interpersonales y el cambio social; incluso su vinculación a los procesos formativos y cognitivos de las personas. Para el ciclista utilitario, la bicicleta ocupa la mayor parte del tiempo dedicado a su movilidad, independientemente de la necesidad: traslado de personas o bienes, acceso a servicios, educación, trabajo, recreación, independientemente del día, hora o condición.

En los territorios de los cantones de Osa, Puntarenas y Cañas, para muchos de los niños la bicicleta se convierte en el eje fundamental para su socialización a través del juego con sus semejantes y la familiarización con el entorno, con el cual interactúan a través de la bicicleta y en el que forman sus lasos y relaciones que posteriormente definen su desarrollo personal. Es común escuchar expresiones como "aquí, antes se aprende a andar en bicicleta que a caminar" (COSEVI, 2012).

En la adolescencia, a través de entrevistas con jóvenes locales, se plantea que la bicicleta parece convertirse en un objeto que les permite canalizar los ímpetus de rebeldía e independencia propios de la edad. Aseguran que la bicicleta les permite movilizarse a largas distancias sin depender de otros medios de transporte que requieren dinero o la autorización (y/o acompañamiento) de los adultos: las escapadas al río o las pedaleadas en grupo; la visitas al parque y el cortejo entre pares, son ejemplos de aquello que la bicicleta les permite hacer, consolidándola como una extensión de su personalidad y asimilando su importancia en los procesos formativos.

Es común observar entre los y las jóvenes la personalización de la bicicleta a través de calcomanías, pinturas especiales $\mathrm{u}$ otras técnicas que les ayuden en su diferenciación, pero también a consolidar la construcción de la propia identidad y su reconocimiento entre sus pares. Es así que en el imaginario de las personas de estas comunidades existen las bicicletas que con rasgos "femeninos" y aquellas que con rasgos "masculinos" (pero también están las "neutrales"), que más que relaciones de poder, denotan la extensión al objeto de cualidades culturales de los sujetos, diferenciando sus propiedades en acorde a las necesidades de los individuos por extender su identidad a través de la bicicleta.

La bicicleta es quizás el vehículo más inclusivo que existe: aun y las personalizaciones mencionadas, no genera ningún tipo de barrera social para su uso, como sí existe para otros vehículos (según estadísticas del COSEVI, más del 85\% de conductores acreditados para carros y motocicletas del país son varones), mucho menos limitaciones en cuanto a condición económica, pertenencia étnica u orientación de género.

No se necesitan permisos especiales ni se incluyen restricciones por edad: desde niños de pocos años, hasta adultos mayores octogenarios se pueden observar montando una bicicleta en las calles de Liberia, Cañas, Puntarenas, Ciudad Cortés o Limón. Esta potenciación de la autonomía es un elemento fundamental en la comprensión de la bicicleta como elemento central en los procesos de conformación comunal y cambio cultural.

Pero la bicicleta también evidencia un uso colectivo. El llevar a los amigos, o a la pareja, "en barra", "en ancas", "sobre la manivela", etc., refiere a las posibilidades de uso de este vehículo, que, por un lado, responde a las necesidades de transporte, pero sobre todo, formar parte de las estructuras sociales de la comunidad. Es común ver al ciclista utilitario regresar en grupo del trabajo o el estudio, charlando animadamente y compartiendo los espacios.

Desde una lectura cultural, la bicicleta es un objeto de enculturación que consolida los lazos sociales y se convierte en eje central de las redes de socialización y los procesos no institucionalizados de enseñanza-aprendizaje al estar presente en todos los momentos de la vida de las personas y formar parte inseparable del paisaje social y cultural de las comunidades.

A los ciclistas utilitarios no se les puede considerar como homogéneos o con características determinadas y uniformes, ni mucho menos agruparlos dentro de colectivos específicos. No ven la bicicleta como un vehículo para el traslado o el ejercicio, sino como extensión de sus relaciones en la cotidianidad de su entorno y un objeto que soluciona sus necesidades diarias de movilidad y socialización, cargado de simbolismos culturales.

Por ello, rara vez asocian la bicicleta y su uso con las políticas de restricción en circulación, movilidad, ordenamiento vial o seguridad vial impulsadas desde las instituciones estatales o locales, al contrario, consideran cualquier acción de control sobre las bicicletas por parte de las autoridades, como una violación a su privacidad y un abuso del Estado. 
Para estas comunidades y sus gentes, para cuando se llegaron a construir los actuales sistemas viales y de transporte, la bicicleta ya estaba, ya formaba parte intrínseca de sus procesos históricos de movilidad. Para ellos, la ampliación de la red vial es simplemente un elemento más en la transformación del entorno, al cual la bicicleta se adapta siguiendo los patrones culturales de las necesidades particulares. Este divorcio conceptual y contextual es lo que ha abierto la enorme brecha en poder garantizar su movilidad segura; la bicicleta no es vista como un medio de transporte (aunque lo sea), sino como parte fundamental de sus relaciones cotidianas. Para el ciclista utilitario el uso de la bicicleta no es opcional.

\section{Nuevas tendencias de uso de la bicicleta: el ciclista recreativo}

Aunque no existen estudios específicos, en las investigaciones cualitativas y de observación ya mencionadas, se ha podido documentar el aumento en la última década del uso de la bicicleta con fines específicamente recreativos o de deporte.

Como se ha mencionado, desde la década de 1940 en Costa Rica existe el ciclismo como deporte élite al que se dedican de manera profesional hombres y mujeres. Sin embargo, ha sido una tendencia de los últimos años el aumento de colectivos que usan la bicicleta con fines de esparcimiento y ejercicio de manera metódica y organizada, sin alcanzar niveles de profesionalización o participar en eventos deportivo de élite.

Estos colectivos se caracterizan por estar integrados por personas con cierto poder adquisitivo que les permite la compra de bicicletas de mayor costo económico que el promedio, además del equipamiento especializado para la práctica del ciclismo, incluyendo dispositivos e indumentaria de seguridad (casco, luces, ropa y zapatos especiales, etc.). Suelen hacer grandes recorridos de decenas de kilómetros, tanto sobre carretera como a campo traviesa, mezclando características de montaña y pista, sobre todo en fines de semana, pero no exclusivamente. En general, la bicicleta no forma parte de su cotidianidad ni es un medio de transporte.

Se mueven en grupo, tienen una estructura jerárquica interna, muchos acceden a esquemas organizacionales más formales como las asociaciones deportivas; suelen tener tarifas de ingreso/afiliación, además de cuotas mensuales; responden a liderazgos internos; cuentan con códigos de comportamiento, formales e informales, sobre su circulación en las vías; y suelen integrarse dentro de colectivos más grandes a nivel regional o incluso nacional. Esta estructura les permite tener una mayor presencia en espacios políticos y de toma de decisiones. Su organización les permite tener posturas bastante homogéneas en temas de interés y establecer líneas discursivas o de acción en consenso.

Para la década del 2000, era más común identificar estos grupos recreativos dentro del Valle Central, sin embargo, en el último decenio se han extendido a casi todas las ciudades y comunidades más pobladas del país. También existen en los cantones con predominancia de ciclistas utilitarios como Liberia, Cañas, Osa, Limón, etc. En estas locaciones es común que el ciclista utilitario también tenga una faceta de ciclista recreativo. Muchos dentro de este sector se han convertido en promotores del movimiento que impulsa la bicicleta como opción a la movilidad, sobre todo en los entornos urbanos.

Cabe destacar que para la zona metropolitana del Valle Central, se ha ido extendiendo el grupo de ciudadanos que abogan por el impulso de la bicicleta como medio de transporte alternativo, sin embargo, aun siendo una minoría, para efectos de esta clasificación los tendríamos que ubicar en un intermedio entre el ciclista utilitario y el recreativo, ya que la bicicleta para ellos no trasciende más allá del transporte y siempre es opcional. Se podrían considerar como parte de una fase inicial, transicional hacia el ciclista utilitario, aunque con una mayor abertura hacia las políticas de movilidad segura y uso de dispositivos de seguridad.

\section{CONCLUSIONES}

Este es apenas un planteamiento inicial para generar una mayor discusión y evidenciar los vacíos en la investigación sobre la movilidad de las personas en bicicleta. El principal error de las políticas públicas sobre movilidad, infraestructura vial y seguridad vial, es considerar el uso y usuarios de la bicicleta como algo homogéneo, sin valorar las condiciones específicas de los diversos usuarios. Se suele tomar como referencia al ciclista recreativo, ignorando por completo la complejidad que deviene en el uso de la bicicleta por parte de los ciclistas utilitarios, para quienes este vehículo trasciende la simple funcionalidad de trasladarse.

Si bien en el país se han construido varios kilómetros de infraestructura para ciclistas (Méndez, s.f.), la evidencia ha demostrado que han sido obras con poco o nulo alcance en la resolución del conflicto que deviene de la interacción del parque automotor con ciclistas, y sobre todo con los ciclistas utilitarios. El alcance de esas obras en la disminución de siniestros de tránsito es más bien limitado. 
Parte de las conclusiones de los estudios de movilidad y seguridad vial en los cantones con predominancia de ciclistas utilitarios, es que las obras en seguridad vial no responden a sus necesidades, $\mathrm{o}$, mejor dicho, las nuevas obras llegan a generar necesidades externas que complejizan aún más su relación en el uso de la bicicleta.

El impacto de las obras viales y planificación urbana ejerce presión y aumenta la vulnerabilidad precisamente de estos segmentos de población, para quienes la bicicleta no es una opción para su movilidad, sino un elemento intrínseco a su cotidianidad y el desarrollo de todas las actividades personales, culturales y económicas.

El discurso que predomina en los foros de discusión sobre movilidad y seguridad vial suelen mantener en su perspectiva al ciclista recreativo o a los nuevos ciclistas que se quieren incluir en la movilidad de las grandes urbes como respuesta al congestionamiento vial, que, como se ha descrito, forman parte de colectivos muy diferentes, con características socioculturales, económicas y necesidades muy particulares, lo que a su vez deviene en exposiciones al riesgo en carretera muy diferentes.

$\mathrm{Al}$ no tener presente a este otro sector, el del ciclista utilitario, que es mayoritario, incluso masivo en cantones como Osa, Liberia, Puntarenas, Limón, Siquirres, Cañas, Pococí, entre otros, seguirá generando políticas públicas e intervenciones de alcance mínimo o incluso negativo, tanto en el ordenamiento del espacio como en la prevención de siniestros viales.
Es indispensable que en las políticas públicas, las nuevas obras viales y las obras complementarias en seguridad vial para ciclistas deben generarse a partir de estudios cualitativos y cuantitativos que permitan comprender al ciclista utilitario, sobre todo en aquellos contextos en donde su presencia es masiva.

No escapan a esta análisis, las contradicciones del discurso académico y de las políticas públicas actuales que buscan estimular y rescatar el uso de la bicicleta dentro del Valle Central como opción para una movilidad sostenible y amigable con el entorno, mientras en aquellas localidades en donde la bicicleta ya es parte intrínseca de los esquemas de movilidad, se implementen obras y políticas públicas que más bien restringen y reprimen el uso de la bicicleta, con carreteras cada vez menos amigables y más inseguras con los ciclistas utilitarios o restricciones cada vez más agresivas en el control de su circulación. Y el efecto es claro, aunque no se haya medido cuantitativamente: cada vez más ciclistas utilitarios parecen incitados a cambiar su medio de transporte, muchas veces por una motocicleta.

Es urgente el llamado a la necesidad de incluir este tipo de consideraciones técnicas de orden cualitativo en la toma de decisiones sobre las vías de desarrollo a seguir en las políticas de mejora y ampliación del sistema vial nacional y cantonal.

\section{REFERENCIAS BIBLIOGRÁFICAS}

1. Acuña, V. H. y Molina, I. (1991). Historia económica y social de Costa Rica (1750-1950). 1era ed. San José: Editorial Porvenir.

2. Bijker, W. (1995). Of Bicycles, Bakelites, and bulbs: Toward a Theory of Sociotechnical Change. MIT Press.

3. Cardoso, C. (1976). La formación de la hacienda cafetalera en Costa Rica (siglo XIX). Avances de Investigación. Proyecto de historia social y económica de Costa Rica 1821-1945. San José, No. 4 (1976), pp. 1-61.

4. Carreón, A.; Martínez, A. y Treviño, X. (2011). Manual del ciclista urbano de la Ciudad de México. Editor Bruno Aceves Humana. Secretaría del Medio Ambiente del Distrito Federal, México.

5. Harlihy, D. (2004). Bicycle: The History. Designed by Sonia Shannon by BW\&A Books, Inc. Printed in the United States of America by Quebecor World, Taunton, Mass.

6. Hilje, B. (1991). La colonización agrícola de Costa Rica (1840-1940). San José, Editorial Universidad Estatal a Distancia.

7. Hughes, J. (2012). Análisis histórico de la participación femenina en ciclismo en Costa Rica y en el mundo. Trabajo Final de Graduación.

8. European Communities. (1999). Cycling: the way ahead for towns and cities. Luxembourg: Office for Official Publications of the European Communities, Belgium.

9. Fletsberaad. (2010). The bicycle capitals of the world: Amsterdam and Copenhagen. Fetsberaad Expertise Centre for Cycling Policy, The Netherlands.

10. Fonseca Herrera, Z. (1979). Los chinos en Costa Rica en el siglo XIX. San José, tesis de Licenciatura en historia, Escuela de Historia y Geografía, Facultad de Ciencias Sociales, Universidad de Costa Rica. 
11. Fonseca Herrera, Z. (2016). Las migraciones chinas a Costa Rica en el siglo XIX. En Historia comparada de las migraciones en las Américas, Ed. Galeana de Valadés. México: UNAM, Instituto de Investigaciones Jurídicas.

12. Fumero, P. (2000). Vida cotidiana en el Valle Central: 1850-1914. Los cambios asociados con la expansión del café. Ana María Botey (coord). Costa Rica. Desde las sociedades autóctonas hasta 1914. San José: Editorial de la Universidad de Costa Rica: pp. 303-338.

13. Instituto para Políticas para el Transporte y el Desarrollo (IDTP). (2012). Planes Integrales de Movilidad: Lineamientos para una movilidad urbana sustentable. Instituto de Políticas para el Transporte y el Desarrollo, México.

14. Loría Chaves, M. (2000). Los inmigrantes chinos dentro de la comunidad costarricense (1870-1910). Tesis de Licenciatura en historia, Facultad de Ciencias Sociales: Universidad de Costa Rica.

15. Méndez Hernández, H. (sf). Estudio de Impacto de Ciclovías en Costa Rica: Promoviendo sistemas alternativos y articulados de transporte urbano en Costa Rica. Fundación para el desarrollo urbano, FUDEU.

16. Molina, I. (2002). Costarricense por dicha. Identidad nacional y cambio cultural en Costa Rica durante los siglos XIX y XX. San José, Editorial EUCR.

17. Morales, G. (1993). Cultura oligárquica y nueva intelectualidad en Costa Rica: 1880-1914. Heredia, Editorial EUNA.

18. Quesada, F. (2007). La modernización entre cafetales: San José, Costa Rica, 1880-1930. Renvall Institute for Area and Cultural Studies, University of Helsinki, Finland.

19. Sáenz García, R.M. (2014). La bicicleta y sus orígenes en Europa. Posgrado de Antropología. Universitat Jaume I de Castello.

20. Salazar, O, (1990). El apogeo de la república liberal en Costa Rica 1870-1914. San José, Editorial de la Universidad de Costa Rica, 1990.

21. Sánchez, R. (1991). Campeonatos nacionales de ciclismo, primera división 1940-1990. San José, C.R.: VASA.

\section{INFORMES FINALES}

22. Consejo de Seguridad Vial (COSEVI). (2012). Seguridad Vial: construcción histórica, redes sociales y contexto sociocultural del cantón de Osa, Pacífico Sur. Informe Final, Bohián Pérez Stéfanov, Inv., Área de Investigación y Estadísticas, COSEVI.

23. Consejo de Seguridad Vial (COSEVI). (2013). Seguridad Vial en zonas costeras, el caso de Puntarenas. Informe Final, Bohián Pérez Stéfanov, Inv., Área de Investigación y Estadísticas, COSEVI.

24. Consejo de Seguridad Vial (COSEVI). (2013a). Seguridad Vial: construcción histórica, turismo y contexto sociocultural del cantón de San Carlos, Zona Norte. Informe Final, Bohián Pérez Stéfanov, Inv., Área de Investigación y Estadísticas, Dirección de Proyectos, Consejo de Seguridad Vial.

25. Consejo de Seguridad Vial (COSEVI). (2013b). La Independencia en dos ruedas, 15 de septiembre, 2013. Informe de actividad, Área de Investigación y Estadísticas, COSEVI.

26. Consejo de Seguridad Vial (COSEVI). (2014). Análisis de riesgos y debilidades en la gestión de la Seguridad Vial en la ampliación de la carretera Interamericana Norte, eje Cañas - Liberia. Informe Final, Bohián Pérez Stéfanov, Inv., Área de Investigación y Estadísticas, COSEVI.

27. Consejo de Seguridad Vial (COSEVI). (2016). Construcción y análisis de perfiles de riesgo en Seguridad Vial y movilidad segura de la Ruta 32: estado actual y escenarios ante una posible ampliación entre la intersección con la ruta nacional 4 y el centro de Limón. Informe Final, Bohián Pérez Stéfanov, Inv., Área de Investigación y Estadísticas, COSEVI.

28. Consejo de Seguridad Vial (COSEVI). (2017). Evaluación desde la Seguridad Vial del proceso de gestión del proyecto de ampliación de la Ruta 1, tramo Cañas - Liberia. Informe Final, Bohián Pérez Stéfanov, Inv., Área de Investigación, Dirección de Proyectos, Consejo de Seguridad Vial. 